\title{
Magnetic monopole loops supported by a meron pair as the quark confiner
}

\author{
Kei-Ichi Kondo* \\ Department of Physics, Graduate School of Science, Chiba University, Chiba 263-8522, Japan \\ E-mail: kondokefaculty.chiba-u.jp
}

\begin{abstract}
We give a definition of gauge-invariant magnetic monopoles in Yang-Mills theory without using the Abelian projection due to 't Hooft. They automatically appear from the Wilson loop operator. This is shown by rewriting the Wilson loop operator using a non-Abelian Stokes theorem. The magnetic monopole defined in this way is a topological object of co-dimension 3, i.e., a loop in four-dimensions. We show that such magnetic loops indeed exist in four-dimensional Yang-Mills theory. In fact, we give an analytical solution representing circular magnetic monopole loops joining a pair of merons in the four-dimensional Euclidean SU(2) Yang-Mills theory. This is achieved by solving the differential equation for the adjoint color (magnetic monopole) field in the two-meron background field within the recently developed reformulation of the Yang-Mills theory. Our analytical solution corresponds to the numerical solution found by Montero and Negele on a lattice. This result strongly suggests that a meron pair is the most relevant quark confiner in the original Yang-Mills theory, as Callan, Dashen and Gross suggested long ago.
\end{abstract}

8th Conference Quark Confinement and the Hadron Spectrum

September 1-6 2008

Mainz, Germany

${ }^{*}$ This work is financially supported by Grant-in-Aid for Scientific Research (C) 18540251 from Japan Society for the Promotion of Science (JSPS). 


\section{Wilson loop and magnetic monopole}

For a closed loop $C$, the Wilson loop operator for SU(2) Yang-Mills connection is defined by

$$
W_{C}[\mathbf{A}]:=\operatorname{tr}\left[\mathscr{P} \exp \left\{i g \oint_{C} d x^{\mu} \mathbf{A}_{\mu}(x)\right\}\right] / \operatorname{tr}(\mathbf{1}), \quad \mathbf{A}_{\mu}(x)=\mathbf{A}_{\mu}^{A}(x) \sigma^{A} / 2 .
$$

The path-ordering $\mathscr{P}$ is removed by using the Diakonov-Petrov version [1] of a non-Abelian Stokes theorem for the Wilson loop operator: in the $J$ representation of $\mathrm{SU}(2)(J=1 / 2,1,3 / 2,2, \cdots)$

$$
\begin{aligned}
W_{C}[\mathbf{A}] & :=\int d \mu_{\Sigma}(U) \exp \left\{i J g \int_{\Sigma: \partial \Sigma=C} d S^{\mu v} f_{\mu v}\right\}, \text { no path-ordering } \\
f_{\mu v}(x) & :=\partial_{\mu}\left[\mathbf{A}_{v}^{A}(x) \boldsymbol{n}^{A}(x)\right]-\partial_{v}\left[\mathbf{A}_{\mu}^{A}(x) \boldsymbol{n}^{A}(x)\right]-g^{-1} \varepsilon^{A B C} \boldsymbol{n}^{A}(x) \partial_{\mu} \boldsymbol{n}^{B}(x) \partial_{v} \boldsymbol{n}^{C}(x), \\
n^{A}(x) \sigma^{A} & :=U^{\dagger}(x) \sigma^{3} U(x), \quad U(x) \in S U(2) \quad(A, B, C \in\{1,2,3\}),
\end{aligned}
$$

where $d \mu_{\Sigma}(U)$ is the product measure of an invariant measure on $\mathrm{SU}(2) / \mathrm{U}(1)$ over $\Sigma$ :

$$
d \mu_{\Sigma}(U):=\prod_{x \in \Sigma} d \mu(U(x)), \quad d \mu(U(x))=\frac{2 J+1}{4 \pi} \delta\left(\boldsymbol{n}^{A}(x) \boldsymbol{n}^{A}(x)-1\right) d^{3} \boldsymbol{n}(x)
$$

where we have introduced a unit vector field $\boldsymbol{n}(x)$.

The geometric and topological meaning of the Wilson loop operator was given in [2]:

$$
\begin{gathered}
W_{C}[\mathscr{A}]=\int d \mu_{\Sigma}(U) \exp \left\{i J g\left(\Xi_{\Sigma}, k\right)+i J g\left(N_{\Sigma}, j\right)\right\}, \quad C=\partial \Sigma \\
k:=\delta^{*} f={ }^{*} d f, \quad \Xi_{\Sigma}:=\delta^{*} \Theta_{\Sigma} \triangle^{-1} \leftarrow \quad \text { (D-3)-forms } \\
j:=\delta f, \quad N_{\Sigma}:=\delta \Theta_{\Sigma} \triangle^{-1} \leftarrow \quad 1 \text {-forms (D-indep.) } \\
\Theta_{\Sigma}^{\mu v}(x)=\int_{\Sigma} d^{2} S^{\mu v}(x(\sigma)) \delta^{D}(x-x(\sigma)),
\end{gathered}
$$

where $k$ and $j$ are gauge invariant and conserved currents, $\delta k=0=\delta j$. Thus, we do not need to use the Abelian projection proposed by 't Hooft [3] to define magnetic monopoles in YangMills theory! The Wilson loop operator knows the (gauge-invariant) magnetic monopole! Then the magnetic monopole is a topological object of co-dimension 3. In $D$ dimensions,

$\mathrm{D}=3$ : 0-dimensional point defect $\rightarrow$ magnetic monopole of Wu-Yang type

$\mathrm{D}=4$ : 1-dimensional line defect $\rightarrow$ magnetic monopole loop (closed loop)

For $D=3$,

$$
k(x)=\frac{1}{2} \varepsilon^{j k \ell} \partial_{\ell} f_{j k}(x)=\rho_{m}(x)
$$

denotes the magnetic charge density at $x$, and

$$
\Xi_{\Sigma}(x)=\Omega_{\Sigma}(x) /(4 \pi)
$$

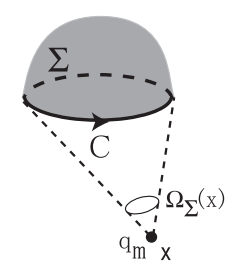

agrees with the (normalized) solid angle at the point $x$ subtended by the surface $\Sigma$ bounding the Wilson loop $C$. Then the magnetic part $W_{\mathscr{A}}^{m}$ is written as

$$
W_{\mathscr{A}}^{m}:=\exp \left\{i J g\left(\Xi_{\Sigma}, k\right)\right\}=\exp \left\{i J g \int d^{3} x \rho_{m}(x) \frac{\Omega_{\Sigma}(x)}{4 \pi}\right\} .
$$

The magnetic charge $q_{m}$ obeys the Dirac-like quantization condition:

$$
q_{m}:=\int d^{3} x \rho_{m}(x)=4 \pi g^{-1} n \quad(n \in \mathbb{Z}) .
$$


The proof follows from a fact that the non-Abelian Stokes theorem does not depend on the surface $\Sigma$ chosen for spanning the surface bounded by the loop $C$. See [2].

For an ensemble of point-like magnetic charges: $k(x)=\sum_{a=1}^{n} q_{m}^{a} \delta^{(3)}\left(x-z_{a}\right)$, we have

$$
W_{\mathscr{A}}^{m}=\exp \left\{i J \frac{g}{4 \pi} \sum_{a=1}^{n} q_{m}^{a} \Omega_{\Sigma}\left(z_{a}\right)\right\}=\exp \left\{i J \sum_{a=1}^{n} n_{a} \Omega_{\Sigma}\left(z_{a}\right)\right\}, \quad n_{a} \in \mathbb{Z} .
$$

The magnetic monopoles in the neighborhood of the Wilson surface $\Sigma\left(\Omega_{\Sigma}\left(z_{a}\right)= \pm 2 \pi\right)$ contribute to the Wilson loop

$$
W_{\mathscr{A}}^{m}=\prod_{a=1}^{n} \exp \left( \pm i 2 \pi J n_{a}\right)=\left\{\begin{array}{ll}
\prod_{a=1}^{n}(-1)^{n_{a}} & (J=1 / 2,3 / 2, \cdots) \\
=1 & (J=1,2, \cdots)
\end{array} .\right.
$$

This enables us to explain the $N$-ality dependence of the asymptotic string tension. See, [4].

For $D=4, \Omega_{\Sigma}^{\mu}(x)$ is the $D=4$ solid angle and the magnetic part reads

$$
W_{\mathscr{A}}^{m}=\exp \left\{i J g \int d^{4} x \Omega_{\Sigma}^{\mu}(x) k^{\mu}(x)\right\} .
$$

Suppose the existence of an ensemble of magnetic monopole loops $C_{a}^{\prime}$ in $D=4$ Euclidean space, $k^{\mu}(x)=\sum_{a=1}^{n} q_{m}^{a} \oint_{C_{a}^{\prime}} d y_{a}^{\mu} \delta^{(4)}\left(x-x_{a}\right), \quad q_{m}^{a}=4 \pi g^{-1} n_{a}$. Then the Wilson loop operator reads

$$
W_{\mathscr{A}}^{m}=\exp \left\{i J g \sum_{a=1}^{n} q_{m}^{a} L\left(\Sigma, C_{a}^{\prime}\right)\right\}=\exp \left\{4 \pi J i \sum_{a=1}^{n} n_{a} L\left(\Sigma, C_{a}^{\prime}\right)\right\}, \quad n_{a} \in \mathbb{Z},
$$

where $L\left(\Sigma, C^{\prime}\right)$ is the linking number between the surface $\Sigma$ and the curve $C^{\prime}$ :

$$
L\left(\Sigma, C^{\prime}\right):=\oint_{C^{\prime}} d y^{\mu}(\tau) \Xi_{\Sigma}^{\mu}(y(\tau)) .
$$

Here the curve $C^{\prime}$ is identified with the trajectory $k$ of a magnetic monopole and the surface $\Sigma$ with the world sheet of a hadron (meson) string for a quark-antiquark pair.

The Wilson loop operator is a probe of the gauge-invariant magnetic monopole defined in our formulation. Thus, calculating the Wilson loop average reduces to the summation over the magnetic monopole charge $(D=3)$ or current $(D=4)$ with a geometric factor, the solid angle $(D=3)$ or linking number $(\mathrm{D}=4)$.

\section{Main results (Magnetic loops indeed exist in $\mathrm{YM}_{4}$ )}

We can show that the gauge-invariant magnetic loop (assumed in the above) indeed exists in SU(2) Yang-Mills theory in $D=4$ Euclidean space: we give a first* (exact) analytical solution representing circular magnetic monopole loops joining two merons $[5] .^{1}$

Our method reproduces also the previous results based on MAG (MCG) and LAG:

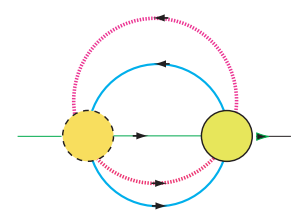

(i) The magnetic straight line can be obtained in the one-instanton or one-meron background. [6]

(ii) The magnetic closed loop can NOT be obtained in the one-instanton background. [8, 9

\footnotetext{
${ }^{1}$ There is an exception: Bruckmann \& Hansen, hep-th/0305012, Ann.Phys.308, 201 (2003). However, it has $Q_{P}=\infty$
} 


\section{Reformulating Yang-Mills theory in terms of new variables}

SU(2) Yang-Mills theory

written in terms of $\mathbf{A}_{\mu}^{A}(x)(A=1,2,3) \quad$ change of variables
A reformulated Yang-Mills theory written in terms of new variables:

We introduce a "color field" $\mathbf{n}(x)$ of unit length with three components

$$
\mathbf{n}(x)=\left(n_{1}(x), n_{2}(x), n_{3}(x)\right), \quad \mathbf{n}(x) \cdot \mathbf{n}(x)=n_{A}(x) n_{A}(x)=1
$$

The color field $\mathbf{n}(x)$ is identified with $\mathbf{n}(x)$ in (1.2). New variables $\boldsymbol{n}^{A}(x), c_{\mu}(x), \mathbf{X}_{\mu}^{A}(x)$ should be given as functionals of the original $\mathbf{A}_{\mu}^{A}(x)$. The off-shell Cho-Faddeev-Niemi-Shabanov decomposition [10] is reinterpreted as change of variables from $\mathbf{A}_{\mu}^{A}(x)$ to $\boldsymbol{n}^{A}(x), c_{\mu}(x), \mathbf{X}_{\mu}^{A}(x)$ via the reduction of an enlarged gauge symmetry. See [11, 12]. Expected role of the color field: 1) The color field $\boldsymbol{n}(x)$ plays the role of recovering color symmetry which will be lost in the conventional approach, e.g., in the MA gauge. 2) The color field $\boldsymbol{n}(x)$ carries topological defects responsible for non-perturbative phenomena, e.g., quark confinement.

\section{Bridge between $\mathbf{A}_{\mu}(x)$ and $\mathbf{n}(x)$}

For a given Yang-Mills field $\mathbf{A}_{\mu}(x)$, the color field $\mathbf{n}(x)$ is obtained by solving the reduction differential equation (RDE): 12

$$
\mathbf{n}(x) \times D_{\mu}[\mathbf{A}] D_{\mu}[\mathbf{A}] \mathbf{n}(x)=\mathbf{0} .
$$

For a given $\mathrm{SU}(2)$ Yang-Mills field $\mathbf{A}_{\mu}(x)=\mathbf{A}_{\mu}^{A}(x) \frac{\sigma_{A}}{2}$, look for unit vector fields $\mathbf{n}(x)$ such that $-D_{\mu}[\mathbf{A}] D_{\mu}[\mathbf{A}] \mathbf{n}(x)$ is proportional to $\mathbf{n}(x)$ : an eigenvalue-like form:

$$
-D_{\mu}[\mathbf{A}] D_{\mu}[\mathbf{A}] \mathbf{n}(x)=\lambda(x) \mathbf{n}(x) \quad(\lambda(x) \geq 0)
$$

The solution is not unique. We choose the solution giving the smallest value of the reduction functional $F_{\mathrm{rc}}$ which agrees with the integral of the scalar function $\lambda(x)$ over $\mathbb{R}^{D}$ :

$$
\begin{aligned}
F_{\mathrm{rc}} & =\int d^{D} x \frac{1}{2}\left(D_{\mu}[\mathbf{A}] \mathbf{n}(x)\right) \cdot\left(D_{\mu}[\mathbf{A}] \mathbf{n}(x)\right)=\int d^{D} x \frac{1}{2} \mathbf{n}(x) \cdot\left(-D_{\mu}[\mathbf{A}] D_{\mu}[\mathbf{A}] \mathbf{n}(x)\right) \\
\Longrightarrow F_{\mathrm{rc}}^{*} & =\int d^{D} x \frac{1}{2} \mathbf{n}(x) \cdot \lambda(x) \mathbf{n}(x)=\int d^{D} x \frac{1}{2} \lambda(x) .
\end{aligned}
$$

\section{Conclusion and discussion}

For given one-instanton and two-meron background $\mathbf{A}_{\mu}(x)$, we have solved the RDE for the color field $\mathbf{n}(x)$ [12]. In the four-dimensional Euclidean SU(2) Yang-Mills theory, we have given a first analytical solution representing circular magnetic monopole loops $k_{\mu}$ which go through a pair of merons (with a unit topological charge) with non-trivial linking with the Wilson surface $\Sigma$.

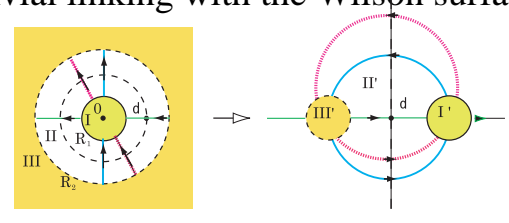


This is achieved by solving the reduction differential equation for the adjoint color (magnetic monopole) field in the two-meron background field using the recently developed reformulation of the Yang-Mills theory [11, 12] and a non-Abelian Stokes theorem [2].

Our analytical solution corresponds to a numerical solution found on a lattice by Montero and Negele [13].

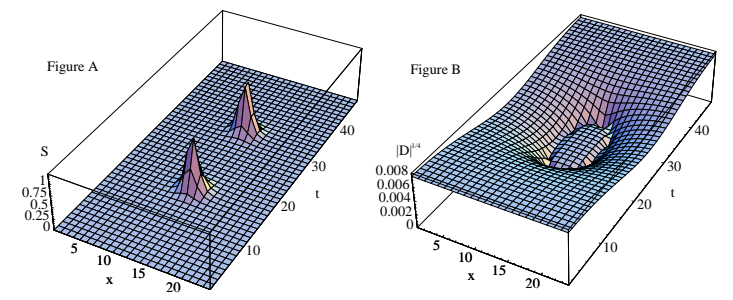

We have not yet obtained the analytic solution representing magnetic loops connecting 2instantons, which were found in the numerical way by Reinhardt \& Tok [7].

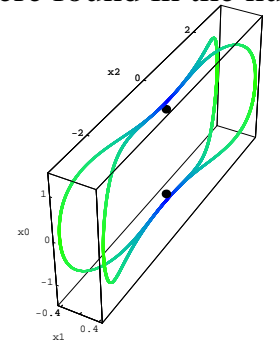

Thus we are lead to a conjecture: A meron pair is the most relevant quark confiner in the original Yang-Mills theory, as Callan, Dashen and Gross suggested long ago [14]. This means a duality relation:

dual Yang-Mills: magnetic monopole loops $\Longleftrightarrow$ original Yang-Mills: merons

\section{References}

[1] D. Diakonov \& V. Petrov, Phys. Lett. B224, 131 (1989); hep-th/9606104.

[2] K.-I. Kondo, arXiv:0801.1274, Phys.Rev.D77, 085029 (2008). K.-I. Kondo, hep-th/0009152.

[3] G. 't Hooft, Nucl. Phys. B190, 455 (1981).

[4] K.-I. Kondo, arXiv:0802.3829, J.Phys.G35, 085001 (2008).

[5] K.-I. Kondo, N. Fukui, A. Shibata \& T. Shinohara, arXiv:0806.3913, Phys. Rev. D78, 065033 (2008).

[6] M. Chernodub \& F. Gubarev, hep-th/9506026, JETP Lett. 62, 100 (1995).

[7] H. Reinhardt \& T. Tok, hep-th/0011068, Phys.Lett.B505, 131 (2001).

[8] R.C. Brower, K.N. Orginos \& C-I. Tan, hep-th/9610101, Phys.Rev.D 55, 6313 (1997).

[9] F, Bruckmann, T. Heinzl, T. Vekua \& A. Wipf, hep-th/0007119, Nucl.Phys.B593, 545 (2001).

[10] Y. M. Cho, Phys. Rev. D 21, 1080 (1980); 23, 2415 (1981); L. D. Faddeev and A. J. Niemi, Phys. Rev. Lett. 82, 1624 (1999); Nucl. Phys. B776, 38 (2007); S.V. Shabanov, Phys. Lett. B 458, 322 (1999); Phys. Lett. B463, 263 (1999).

[11] K.-I. Kondo, T. Murakami \& T. Shinohara, hep-th/0504107, Prog. Theor. Phys. 115, 201 (2006).

[12] K.-I. Kondo, T. Shinohara \& T. Murakami, arXiv:0803.1076, Prog. Theor. Phys. 120,1 (2008).

[13] A. Montero \& J.W. Negele, hep-lat/0202023, Phys.Lett.B533, 322 (2002).

[14] C.G. Callan, R. Dashen and D.J. Gross, Phys. Rev. D 17, 2717 (1978). 